\title{
Proposal and evaluation of a future mobile network management mechanism with attractor selection
}

Gen Motoyoshi ${ }^{1,2 *}$, Kenji Leibnitz $^{2,3}$ and Masayuki Murata ${ }^{2}$

\begin{abstract}
Several task forces are currently working on how to design the future Internet and it is high time for research work to also move a step forward to future mobile networks on a large scale. In this article, we propose a future mobile network management method based on a combination of OpenFlow and the biologically inspired attractor selection method to achieve scalability and energy efficiency. In other words, we propose novel approaches to wireless network management by extending the attractor selection mechanism in path and cluster management for signaling cost reduction. First, in path management, we establish a control method that each mobile node selects the best suited interfaces in accordance with instantaneous live traffic volume. Then, in cluster management, we design a network management method that network devices select the best OpenFlow cluster to join in order to reduce handover signaling cost. Through autonomous decisions of each mobile node and network device, the whole wireless network can be managed in an autonomous, energy efficient, and robust manner.
\end{abstract}

\section{Introduction}

We are now facing a new era when the future Internet infrastructure needs to be drastically changed from scratch in order to meet the great variety of requirements from users. In the meantime, there has been an increasing number of research activities on future Internet infrastructures [1] applicable to the field of Information, Communication, and Energy Technology (ICET). All over the world, we can see many research task forces, such as four projects that National Science Foundation (NSF) promotes as the Future Internet Architecture (FIA) [2] in the United States, the European Future Internet Assembly [3] under the European Seventh Framework Program (FP7), and AKARI [4] in Japan, which are all working on future Internet research work. From the viewpoint of user needs for our future society, the wireless communication environment is essential to provide users with mobile services and there have been several research activities on future

\footnotetext{
${ }^{*}$ Correspondence: g-motoyoshi@aj.jp.nec.com

${ }^{1}$ Cloud Systems Research Laboratories, NEC Corporation, 1753 Shimonumabe,

Nakahara-ku, Kawasaki, Kanagawa, 211-8666, Japan

2 Graduate School of Information Science and Technology, Osaka University,

1-5 Yamadaoka, Suita, Osaka 565-0871, Japan

Full list of author information is available at the end of the article
}

wireless networks such as the MobilityFirst Project [5] of the FIA program and Programmable Open Mobile Internet (POMI) 2020 of the Stanford Clean Slate Program. In addition, there are several architecture proposals for the future mobile network with separated identifier and locator [6]. Among all these activities, one of the most promising future Internet research activities is OpenFlow technology [7] to construct a programmable and environmentally friendly ICET infrastructure [8-10]. OpenFlow technology has the potential to meet a wide variety of requirements from users due to its programmability and it is available not only for wireless communication but also for wired networks. However, the above research has been limited within local sites such as campus networks and data center networks for the time being and OpenFlow operates in a centralized way which may lead to scalability problems.

In addition to mobility support, robustness is one of the keywords when talking about the future Internet infrastructure for coping with disasters like earthquakes or tsunamis. In addition, energy saving for protection of the environment is also an important factor. To achieve the above targets, more reliable infrastructures based on scientific theory are preferred over approaches that are

\section{是 Springer}

(c) 2012 Motoyoshi et al: licensee Springer. This is an Open Access article distributed under the terms of the Creative Commons Attribution License (http://creativecommons.org/licenses/by/2.0), which permits unrestricted use, distribution, and reproduction in any medium, provided the original work is properly cited. 
simply derived from empirical experience. For instance, biological systems have evolved over a long period of time to exhibit an internal robustness against environmental changes, which helps in the survival of the species. For this reason, there have been recently several mechanisms based on biological systems applied to future ICET [1113]. These biologically inspired mechanisms achieve a good overall system construction from several viewpoints such as performance and robustness despite changes of the operating environment. Such condition changes occur frequently especially in wireless communication networks and therefore applying mechanisms from biology is very promising. Among biologically inspired control mechanisms, attractor selection is one of the possibilities to formulate a mathematical model based on biological dynamics. Recently, there have been several research activities applying the attractor selection method to various ICET management fields in the Yuragi Project [14] and in the Global Center of Excellence Program for Founding Ambient Information Society Infrastructure [15] in Japan.

As a societal network infrastructure of the future, it is of great value to achieve scalability and robustness in a well-balanced manner at the same time. The future mobile network and the above-mentioned attractor selection method have so far been well investigated independently of each other. However, the combination of both has not been studied yet. In this article, we extend the above attractor selection mechanism in order to work on large-scale mobile wireless network environments based on OpenFlow technology. Our contribution in this article is as follows. First, we discuss the future mobile network on the basis of a combination of OpenFlow technology and the attractor selection method from a general perspective. Second, we propose a concrete method for a mobile node to select the best radio interfaces under varying environmental conditions with attractor selection driven by realtime user traffic volume. Third, we establish an appropriate clustering method to reduce handover signaling cost on OpenFlow-based future mobile networks with attractor selection driven by the difference of the flow directions between user traffic and signaling traffic. Finally, we evaluate our proposed multi-radio interface selection mechanism by simplified computer simulations. Simulation results show that our proposed mechanisms are feasible enough to work in the presence of fluctuations on the radio channel and in data traffic volume. Our extended attractor selection model has an ability to select environmentally optimized radio and network resources irrespective of environmental changes. As stated above, our contribution will offer a biologically inspired and robust optimization method in a self-organizing manner especially for wireless communication networks.

The rest of this article is organized as follows. We first discuss some related work in the following section. In
Section "Research background and problem statement" we explain the basic mechanism of attractor selection and discuss general issues on the adaptation of the attractor selection method into an OpenFlow-based future mobile network. In Section "Proposed extension of attractor selection model", novel concepts to adopt the attractor selection mechanism into the future mobile network environment are proposed and discussed in detail. In addition, in Section "Evaluation of attractor selection extension", simulation results are explained. Finally, in the last section, some conclusions are given.

\section{Related study}

The current Internet has evolved to maintain a backward compatibility by adding new functions whenever they are needed. However, a consensus was reached among research leaders from academia and industry that the new future Internet infrastructure should be redesigned from scratch. One key technology for the future Internet is network virtualization established by programmable network components based on OpenFlow technology [8]. As an essential fraction of the future Internet, Yap et al. [9] drew a blueprint for future wireless mobile networks that can achieve handovers between WiMAX and WiFi environments. In addition, Yap et al. [10] deployed a testbed named as OpenRoads to offer a slicing service in wireless infrastructure on a campus network. Here, slicing service means that shared common wireless resources can be offered to users in a flexibly separated manner at a fine granularity. However, these studies are limited to localized areas like data centers and campus networks.

For utilizing multiple radio interfaces, cognitive radio technologies [16] are promising and have been well investigated. Focusing on radio interface selection, several vertical handover methods in heterogeneous wireless networks are investigated. Zhu et al. [17] proposed an optimization algorithm of policy-based vertical handover. Merlin et al. [18] discussed a resource allocation method to work on multi-channel multi-interface multi-hop wireless networks. Kassar et al. [19] surveyed vertical handover technologies and analyzed the essence of vertical handover. Most of the current studies are based on precise handover management and it is not sure if they will work on a large scale. In addition, clustering technology is often used for forming localized communication groups to gain scalability in wireless sensor networks (WSN). Yi et al. [20] proposed an energy-efficient clustering algorithm, PEACH (Power-Efficient and Adaptive Clustering Hierarchy protocol). However, Jiang et al. [21] compared existing clustering methods in WSNs and revealed several open issues such as cluster formation in heterogeneous networks, mobility support, and so forth.

From the viewpoint of biologically inspired networks and communications, there have been several research 
outcomes. The articles by Dressler and Akan [12] and Meisel et al. [13] provide good surveys for the large quantity of biologically inspired methods that are currently applied to networking problems. Investigated problems range from ant-based routing in mobile ad hoc networks [22], artificial immune systems for recovering from query losses in sensor networks [23], homeostatic regulation of the blood glucose level as IP resource self-management method [24] to entire architectures operating according to biological principles $[25,26]$.

Our goal in this article is to extend OpenFlow technology with the robustness occurring in biological systems. To achieve this goal, we apply the attractor selection method as adaptive and robust control mechanism in OpenFlow. In its original context within biological systems, Kashiwagi et al. [27] formulated a mathematical model of attractor selection to express an adaptive response system in the dynamics of gene expression in Escherichia coli cells. This dynamic behavior is formulated through differential equations of mRNA concentrations in a cell considering the environmental changes of nutrient conditions under the influence of noise. Special stable states exist in this dynamic system as attractors and once the system state has approached an attractor it will remain there. On the other hand, if the environment changes that this attractor becomes unstable, the inherent noise will drive the system state to a new stable attractor. This switching between convergence and search phases is controlled by an activity term, which corresponds to the cell's growth rate. In other words, the activity locks the system to stay at the same attractor and noise works for offering the trigger to find the other attractor. Based on the above models, Murata [15] developed and extended ambient network management to facilitate future human life closely related to environmental adaptability. Several research activities on the attractor selection have been produced within the Yuragi Project ${ }^{a}[14]$ and by other researchers.

Within the framework of the above projects Leibnitz et al. [28] extended the Kashiwagi-model to a multidimensional control mechanism and showed an instance of attractor selection applied to multi-path routing in ad hoc networks. Wakamiya et al. [29] surveyed a wide variety of biologically inspired systems and built a scalable architecture focusing on systems running in a selforganizing and autonomous manner based on attractor selection. Furthermore, Leibnitz and Murata [30] analyzed the perturbation effects in attractor selection based on observations of the system's responsiveness to inherent fluctuations. Kajioka et al. [31] applied an attractor selection model to a multi-interface selection system using several wireless media such as LTE, WiMAX, and WiFi with different communication capabilities. Koizumi et al. [32] adopted an attractor selection mechanism into virtual network topology construction. The best overlay network topology at each moment is selected according to the outputs of the attractor selection equation. $\mathrm{Li}$ et al. [33] utilized attractor selection in wavelength division multiplexing mesh networks to execute intentional path reroutes especially for unpredictable future resource demands.

In addition, attractor selection has been investigated in a wide variety of fields beside data communication. Chujo et al. [34] showed that attractor selection is applicable for a real-time production scheduling. Fukuyori et al. [35] proposed a control method of a human-like robot arm with attractor selection and confirmed that the method is feasible enough to work just with simple feedbacks and without a global knowledge of the robot. Kitajima et al. [36] achieved construction of a data broadcasting service with the filtering order decision based on an attractor selection. All these studies demonstrate that attractor selection is feasible as a simple and robust control scheme that can be applied to various types of optimization and scheduling problems.

\section{Research background and problem statement}

This section first introduces a basic attractor selection model based on Adaptive Response by Attractor Selection [27]. The generalized mathematical model is expressed as follows:

$$
\frac{d m}{d t}=f(m) \times \alpha+\eta
$$

where function $f$ is defined by a potential function and is a function of the state $m, \alpha \in[0,1]$ is an activity of the selected attractor, and $\eta$ is a noise term. At time $t$, one attractor is selected based on the output of Equation (1). Here, the function $f$ determines the attractors through $f(m)=-d U(m) / d m$, where $U(m)$ is the potential function. The activity $\alpha$ indicates the suitability of the selected attractor to the current environment. The more suitable the attractor is, the larger the activity becomes and the attractor basins become deeper which prevents that noise perturbs the state away from this attractor (see Figure 1). Hence, in case of larger $\alpha$, the deterministic function $f$ is dominant over noise to find an attractor. On the other hand, smaller $\alpha$ makes the shape of the potential flatter and the random term $\eta$ becomes dominant which permits that the state can easily leave the current basin. Changes of environmental conditions reflect on the change of activity $\alpha$ appropriately for controlling the system. Hence, depending on the transition of activity $\alpha$, both deterministic mode and stochastic mode are switched over and it is well suited for finding a tradeoff between reducing unnecessary management cost and pursuing the highest 


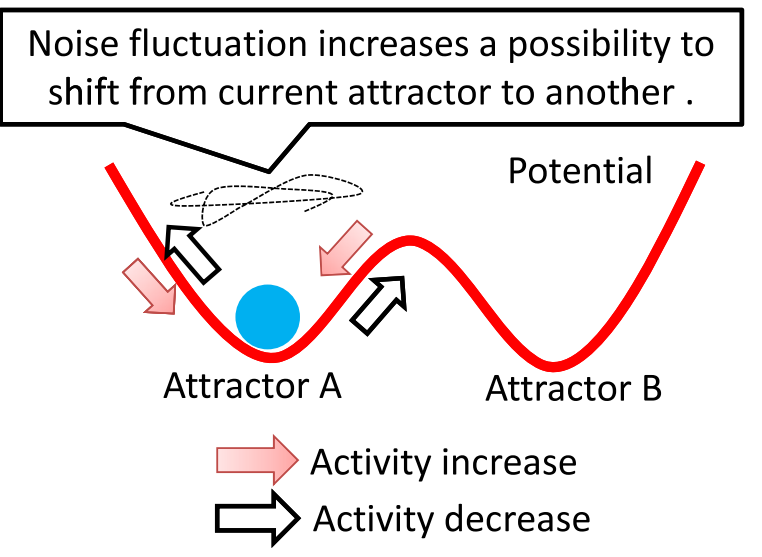

Figure 1 Sketch of basic attractor selection mechanism.

performance. In the end, this attractor selection procedure is an effective way to an adaptive system construction for coping with environmental changes.

In addition, we will discuss our vision of a future mobile network architecture. We focus on a network based on OpenFlow due to many of its strong points such as flexible path management with reference to complicated requirements from users. However, OpenFlow also has some drawbacks. First, energy cost for path calculation increases in return for flexible path establishment. Second, traffic overhead caused by frequent handovers increases because an OpenFlow Controller (OFC) has its own local domain and is not suitable for managing a large scale network as it is. A domain of OpenFlow network consists of a single OFC and several OpenFlow Switches (OFS). The OFC maintains status information such as flow tables of all switches under the controller. Hence, the controller has processing requirements that are proportional to the number of switches in order to carry out flexible flow table management. In general, each local domain of the entire OpenFlow network is independently pre-designed by each network administrator. If a mobile node moves between two domains, each controller has to exchange signaling messages with each other to sustain the communication session of the mobile node. An example of the inter-domain handover sequence in an OpenFlow network is shown in Figure 2. Therefore, lightweight control is essential for the scaling of OpenFlow network management and reduction of inter-domain handover signaling. OpenFlow is a technology where centralized controllers can control user data paths of all network devices such as switches, routers, and base stations through an external standard API. In addition, data packets are handled on a flow basis, and therefore, flexible data path arrangement is possible. However, the centralized basis gives cause for concern about scalability. Hence, we introduce a method based on attractor selection to overcome the above drawbacks.

\section{Proposed extension of attractor selection model}

In this section, we propose a future mobile network architecture taking into account both the flexibility of OpenFlow and the robustness of attractor selection. Our proposed architecture is shown in Figure 3. In this architecture, OFCs manage all OpenFlow devices and simultaneously each device uses attractor selection in an autonomous manner to achieve its own targets such as reduction of energy consumption, end-to-end delay, and so forth. This combination of a strict control by OpenFlow and a loose control by attractor selection is useful to meet a variety of user needs for the future network. Here, each OFC manages all OpenFlow devices that are within its domain. In general, an operator covers a large network area that the network consists of several OFCs and devices. In this article, we assume that the OpenFlow network is managed by a single operator.

In this section, we will propose two approaches to enhance the future mobile network infrastructure by using the attractor selection method. One is the method of multi-interface selection operating on mobile node side and the other is the method of adaptive clustering method working on network side. Both of them contribute to energy saving to protect the environment.

\section{Multi-interface selection of mobile node}

In the future mobile network environment, different wireless media are assumed to be available in a mobile node on cognitive radio infrastructures [16]. The number of access media types, application types, and volume of traffic will increase in a large scale and for this kind of explosion in diversity it is expected that conventional centralized mobility management and mere distributed mobility management cannot work well. As a matter of fact, existing simple vertical handovers are not sufficient enough to efficiently manage the wide variety of radio access technologies to meet the complicated user demands on the future mobile network. There is a need for scalability and robustness and therefore we focus on attractor selection with the capabilities of biologically mechanisms. In future mobile networks, the performance of the best interface for a mobile node might be fluctuating according to environmental changes especially due to diversity. The interface should be selected based on several aspects such as radio quality, traffic distribution, and required QoS. Handling all the conditions by the OFC is inefficient and hence a kind of abstract control mechanism should be installed which can handle any kind of objective.

We chose the well-investigated and reliable attractor selection model from [31] as a basis for our proposal. In [31], the degree of satisfaction is calculated by using 


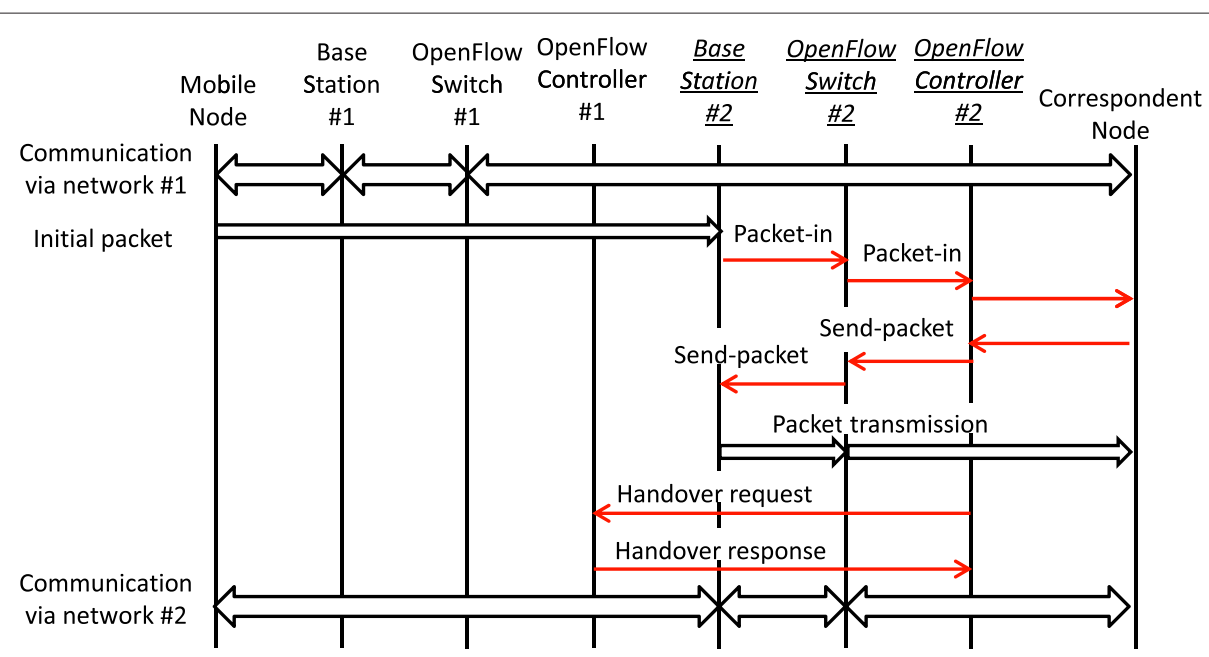

Figure 2 An example of inter-domain handover sequence.

the information of how close pre-defined QoS and live conditions are and therefore the system tends to prefer conditions that are closest to the given target QoS over conditions that are far from the target QoS, but may still be acceptable. This kind of acceptable and qualified better conditions might be avoided in the existing model. In our model, in order to aim for better wireless communication conditions, the activity equation has been extended to work in a more effective manner by using live traffic information instead of passive pre-defined QoS information as in [31]. Our extension contributes to taking care of wider ranges of wireless conditions and hence can easier cope with even unexpected events. This extension permits managing more challenging cases with large environmental changes. Our proposed model is expressed by the following equations.

$$
\begin{aligned}
\frac{d m_{i}}{d t} & =\frac{S(\alpha)}{1+\max \left(m_{i}\right)^{2}-m_{i}^{2}}-D(\alpha) m_{i}+\eta_{i} \\
S(\alpha) & =\alpha\left(\beta \alpha^{\gamma}+1 / \sqrt{2}\right) \\
D(\alpha) & =\alpha \\
\alpha & =\left\{\begin{array}{cc}
1 /(1+\exp (-G Q) & (Q>0) \\
0 & (Q=0)
\end{array}\right. \\
Q & =\left(\overline{\mathrm{SNR}} / \mathrm{SNR}_{*}+\overline{\mathrm{BW}} / \mathrm{BW}_{*}\right) / 2
\end{aligned}
$$

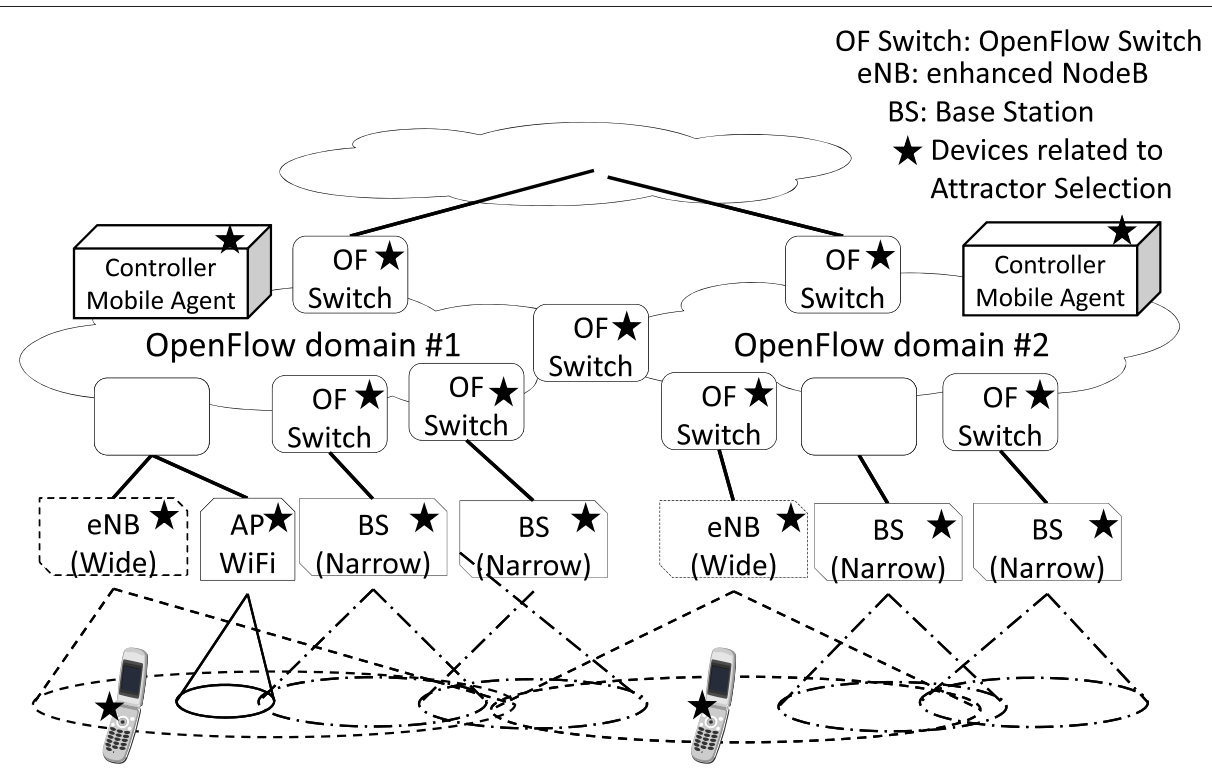

Figure 3 An example of future mobile wireless network. 
Let us assume that a mobile node has $N$ available radio interfaces over which it can connect to the OpenFlow network where $m_{i}$ indicates the selection fitness of each radio interface $i=1, \ldots, N$ and $\eta_{i}$ is white Gaussian noise. $\beta$ and $\gamma$ are system parameters to design the depth of the potential at the attractor and the adaptation strength of activity, respectively. $\alpha \in[0,1]$ is the activity to express suitability of the selected interface for the current environment, which is obtained as the output of a sigmoid function of $Q$ with $G$ as a gain parameter. This gain parameter is used for the adjustment of sensitivity between activity $\alpha$ and environmental parameter $Q$. The environmental parameter on each interface $Q$ is calculated by using realtime signal-to-noise ratio (SNR) and available bandwidth (BW) from the statistics of packet loss, which are shown in Equations (7) and (8), respectively. These two parameters, SNR and BW, are calculated based on realtime information and they therefore reliably reflect the current environmental conditions. Basic equations of attractor selection are introduced from [31] and this mathematical model of SNR and BW are derived from [37]. Here, the parameters with overline and asterisk denote average and target value over the whole samples, respectively. Hence, fluctuating environmental information like packet loss is embedded implicitly in $Q$. This $Q$ indicates the ratio of necessary QoS against live traffic volume on the radio media and is closely related to the activity, which is equivalent to the satisfactory degree of media selection. As SNR and BW both approach toward their respective target values, $Q$ gets closer to 1 . As a result also the activity $\alpha$ approaches 1 , which means that the selected interface is suitable to operate under the current environmental conditions. The dynamics provides us with the selection of the best interfaces, which leads to energy conservation by avoiding unnecessary radio resource consumption and unnecessary signaling cost overhead. In addition, once environmental conditions become inappropriate, the noise effect from $\eta_{i}$ increases and a different radio interface will be randomly selected in an autonomous manner until a sufficient one is found.

$$
\begin{aligned}
& \mathrm{SNR}= 2 \times \operatorname{erfc}^{-1}(2 \times \mathrm{BER})(=\text { realtime SNR }) \\
& \mathrm{BW}= \frac{\text { the volume of successfully received data }}{\text { unit time }} \\
&(=\text { available bandwidth })
\end{aligned}
$$

Here erfc is the complementary error function and its inverse function $\mathrm{erfc}^{-1}$ is expressed as follows with the Taylor expansion.

$$
\begin{aligned}
\mathrm{BER} & =\frac{\text { the volume of successfully received data }}{\text { the volume of transmitted data }} \\
\operatorname{erfc}(x) & =\frac{2}{\sqrt{(} \pi)} \int_{x}^{\infty} \exp ^{-t^{2}} d t \\
\operatorname{erfc}^{-1}(z) & =\sum_{k=0}^{\infty} \frac{c_{k}}{2 k+1}\left(\frac{\sqrt{\pi}}{2} z\right)^{2 k+1}
\end{aligned}
$$

In our proposed mechanism, realtime information such as live wireless communication conditions is utilized to drive the above equation and therefore it is expected that additional artificially induced noise like in [31] is not necessarily if there is already sufficient noise on this live traffic information depending on the quality of wireless communications. This is because noise elements are already included in this model. This removal of artificial noise is expected to lead to complexity reduction in system design for network administrators to achieve maintenance cost reduction and prevent from latent human errors. In addition, in the case of worse wireless conditions, greedy selection based on instantaneous values is not necessary anymore or is even harmful from the viewpoint of system cost. Here, our system based on attractor selection has two modes such as deterministic and stochastic modes. Therefore, we can achieve more efficient system management to select system parameters accurately enough in good radio link conditions (deterministic mode) or loosely enough in noisy radio link conditions (stochastic mode). This is one of our benefits, compared with the other systems that rely on the accurate decision based on the selection of the interface with the best realtime BER.

\section{Adaptive clustering of controller domain}

In addition, in the future mobile network, several types of access edge devices are assumed to appear flexibly responding to user needs. Recently, base stations with small coverage like Femto cells [38] are expected to increase in number owing to the growing need for high data rate transmission for richer services. In addition, cognitive radio [16] is another promising technology to accomplish comfortable communication from the viewpoint of efficient frequency usage. Hence, the mixture of different access technologies is most likely to produce more and more opportunities for both vertical and horizontal handovers to cause large amount of signaling traffic between OFSs and OFCs.

We would like to reduce the above signaling cost caused by handovers and utilize attractor selection in adaptively sustaining appropriate clusters according to environmental dynamics from the viewpoint of signaling cost reduction. Our proposed clustering mechanism works in an autonomous manner over an ordinary OpenFlow network managed in a centralized manner due to the OpenFlow management concept. Here, universality of attractor 
selection is one of the important features and hence the same basic model is used for this solution. On this issue, the same equations as Equations (2-5) described in the previous section are used, where only $m_{i}$ is replaced by $s_{i}$ indicating the selection probability of a cluster group equivalent to each OFC $i$. The network is divided into several network clusters and each cluster is controlled by an OFC. Each domain consists of some OFSs, base stations, and mobile nodes in addition to an OFC. In our proposed model, a mobile node selects one of the clusters by using the attractor selection model. Parameters $\eta_{i}$, $\beta$, and $\gamma$ are kept the same as in previous section. While most of the equations and parameters from the previous section can directly be reused, we need to adapt $\alpha \in[0,1]$ as the activity to express suitability of the selected cluster equivalent to OFC that the device should join. This activity is expressed again as output of a sigmoid function of an environmental parameter and a gain parameter. The environmental parameter of each cluster $s_{i}$ is calculated based on the ratio of user data traffic volume against signaling traffic volume for the path setup on the cluster by using realtime traffic volume passing through the network device. The larger this parameter value is, the more likely the network device should stay at the current cluster. On the contrary, the smaller it is, the more aggressively the network device should change the cluster that it belongs to. Hence, fluctuating environmental information like traffic changes is embedded implicitly on this environmental parameter. This value is closely related to the activity, which is equivalent to the satisfactory degree of cluster selection. Therefore, this activity indicates the ratio of user traffic volume against signaling traffic volume for the path setup, which corresponds to high probability to alleviate frequent handovers. This dynamics provides us with the selection of the best clusters, which leads to cost reduction of frequent handover signaling overhead.

According to attractor-based dynamics, each network device selects the most appropriate cluster equivalent to OFCs. As for attractor selection on the controllers themselves, it would work if the OpenFlow network is established in a hierarchical manner. In this scenario, it is assumed that network devices have knowledge about neighboring or reachable OFCs in advance. In addition, in the future, popularity of always-on devices produces less signaling traffic caused by initial power-on procedure than those caused by handovers due to mobility. An example image of clustering is shown in Figure 4. The network is divided into clusters A and B marked by a dashed and a solid line, respectively. According to the attractor selection model, some devices of cluster A within the oval are assumed to have a high probability of handover and therefore are moved to cluster $\mathrm{B}$. This dynamics is expected to formulate the most appropriate cluster distribution from the viewpoint of the optimal signaling cost by handover, which translates to energy cost. However, we should carefully grasp the tradeoff of reduction of handover cost and increase of cluster switchover cost. Finally, as stated in the previous section, the effect by utilization of intrinsic noise is also expected on this dynamic clustering solution instead of artificial noise.

\section{Evaluation of attractor selection extension}

In this section, we introduce simulation results of multiradio interface selection by attractor selection. We simulate wireless communication based on a code division multiple access channel on a transmission line under frequency selective fading and analyze basic tendencies of our proposed mechanism.

\section{Simulation model of multi-interface selection}

As a simulation model, on the transmitter side, binary data are modulated by Phase Shift Keying, spread by $M$ sequence (maximal length sequence), and transmitted after multiplying the carrier frequency. This $M$ sequence is categorized as Pseudo Noise (PN) code due to its code generation approach. On the receiver side, the signal is decoded after despreading and demodulation. As for the transmission line, a multi-path fading transmission line is assumed. A mobile node has two radio interfaces with different frequency band usage and each interface is influenced by two path Rayleigh fading [37]. In addition, additive white Gaussian noise (AWGN) is added after the fading effect to account for shadow fading.

Under the above-mentioned conditions, we simulated multi-radio interface selection with our proposed attractor selection mechanism. Basic simulation parameters are listed in Table 1. A basic attractor selection function diagram is shown in Figure 5. A selector periodically monitors the statistics of packet transmissions on both radio interfaces and calculates realtime wireless interface SNR and realtime available bandwidth. With them, a "Yuragi controller" selects the best radio interface on that condition.

In addition, the Yuragi-based selection algorithm is shown in Figure 6. In Figure 6, the best interface is first selected at random (Step 1) and packet transmission behaviors are monitored (Step 2). Based on the above information, realtime SNR and realtime available bandwidth are calculated (Steps 3 and 4). After Step 4, Yuragi-based equation (Equation 2) is calculated. According to the output of these calculation results, the best radio interface is selected (Step 5) and it is maintained by iteration of this cycle. This Yuragi-based attractor selection equation has two features at the same time. One feature is that it converges to an attractor when in good wireless communication condition, i.e., when it is easy to estimate environmental conditions accurately. The other feature is to select the candidate in a random manner when in bad 


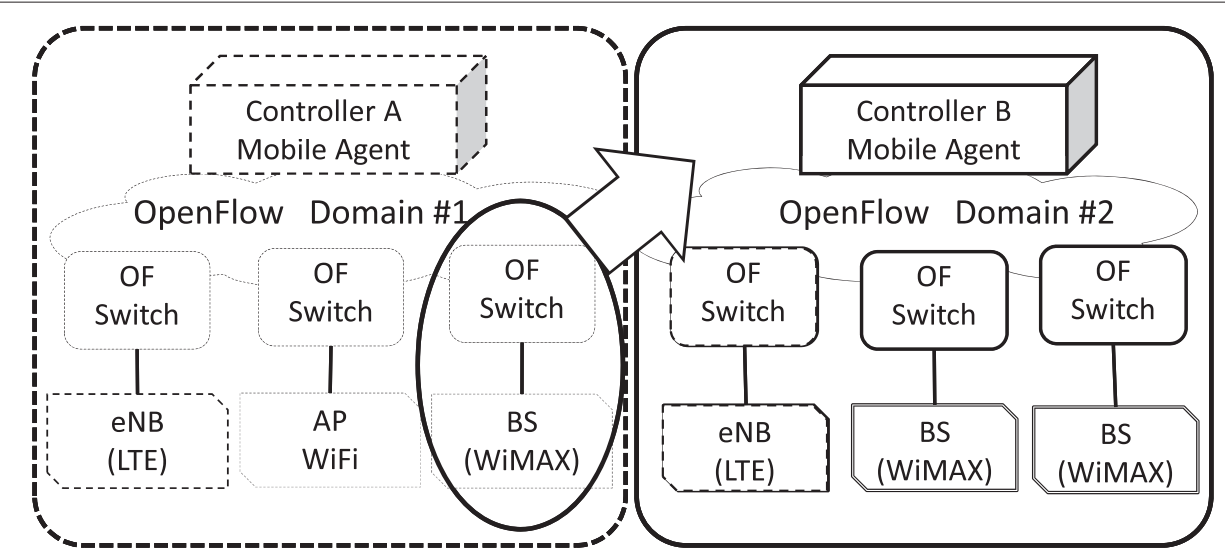

Figure 4 An image of dynamic clustering.

wireless communication condition, which means that it is hard to predict environmental conditions. Hence, attractor selection has a potential to survive on both good and bad environmental conditions. This is a huge difference between attractor selection and merely greedy selection.

\section{Simulation and numerical analysis}

Simulation results are shown in this section for the multiinterface selection problem. Furthermore, we show some numerical evaluation for the clustering case to demonstrate that our proposed clustering method can achieve a lower handover cost, which which shows that it is more energy efficient.

\section{Simulation results of multi-interface selection}

The results are obtained on the condition that the radio link quality on the mobile node's interface varies as shown in Figure 7. We assume the following scenario to investigate the performance of a system with two interfaces. Let us assume that interface \#0 initially has a certain radio

Table 1 Basic simulation parameters in evaluations of multi-interface selection method

\begin{tabular}{ll}
\hline Parameter & Value \\
\hline Number of radio interfaces & 2 \\
Spreading code length & 31 chip \\
Chip rate & $32 \mathrm{Mcps}$ \\
Data transmission rate & $1 \mathrm{Mbps}$ \\
Data decision method & Maximum likelihood decision \\
& $\quad$ (matched filter) \\
Carrier frequency & $20 \mathrm{MHz}$ \\
Transmission line model & AWGN 2-path Rayleigh fading \\
& (Delay=1 chip) \\
Minimum threshold for $E_{b} / N_{0}$ & $10 \mathrm{~dB}$ \\
Minimum threshold for Bandwidth & $100 \mathrm{~Hz}$ \\
\hline
\end{tabular}

link quality of $F_{0 \text {,base }}$ and interface \#1 has $F_{1 \text {,const, which is }}$

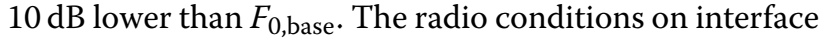
$\# 0$ remain the same until $F_{0 \text {,base }}$ drops by $40 \mathrm{~dB}$ to $F_{0 \text {,down }}$ in the time interval for $t \in[600,1000]$ and recovers again after that to the original value $F_{0 \text {,base }}$. On the other hand, interface \#1 with the initially lower quality maintains it as $F_{1 \text {,const }}$ throughout all 1500 time steps.

The switchover tendencies of the selected active radio interface are shown in Figure 8, which are the results in the case that $F_{0 \text {,base }}$ of the channel is equivalent to $10,20,30$, and $40 \mathrm{~dB}$, respectively. In Figure 8a,b, it is shown that the best radio interface fluctuates frequently between interface $\# 0$ and $\# 1$ in case of 10 and $20 \mathrm{~dB}$, respectively. This is because in both cases initial noise levels are not so high and therefore the noise level shift by $40 \mathrm{~dB}$ as well as the constant noise level difference by $10 \mathrm{~dB}$ between interface $\# 0$ and \#1 do not cause any discrepancy in interface selection performance. In such kind of situations, in order to

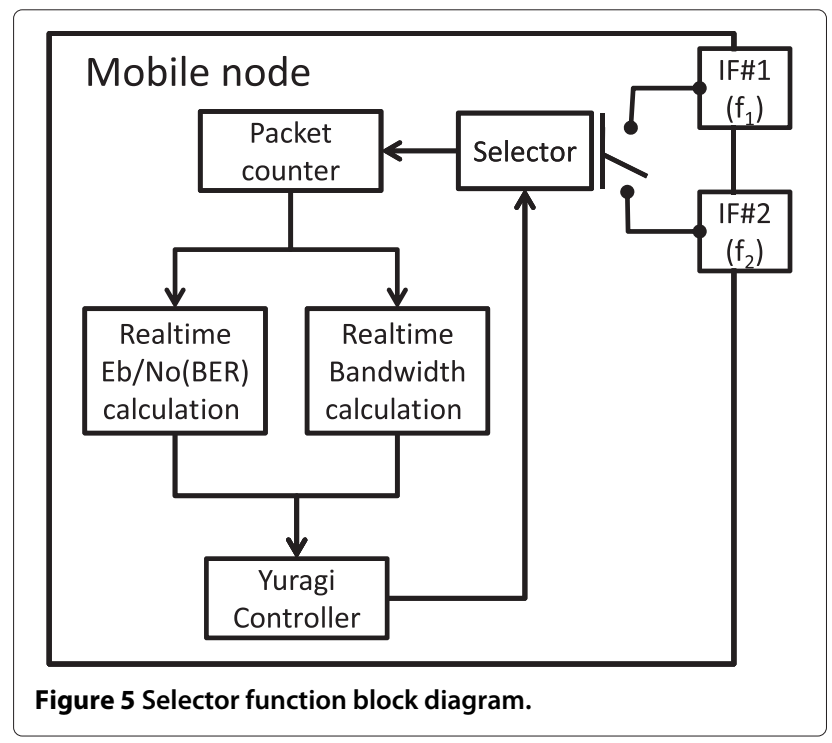




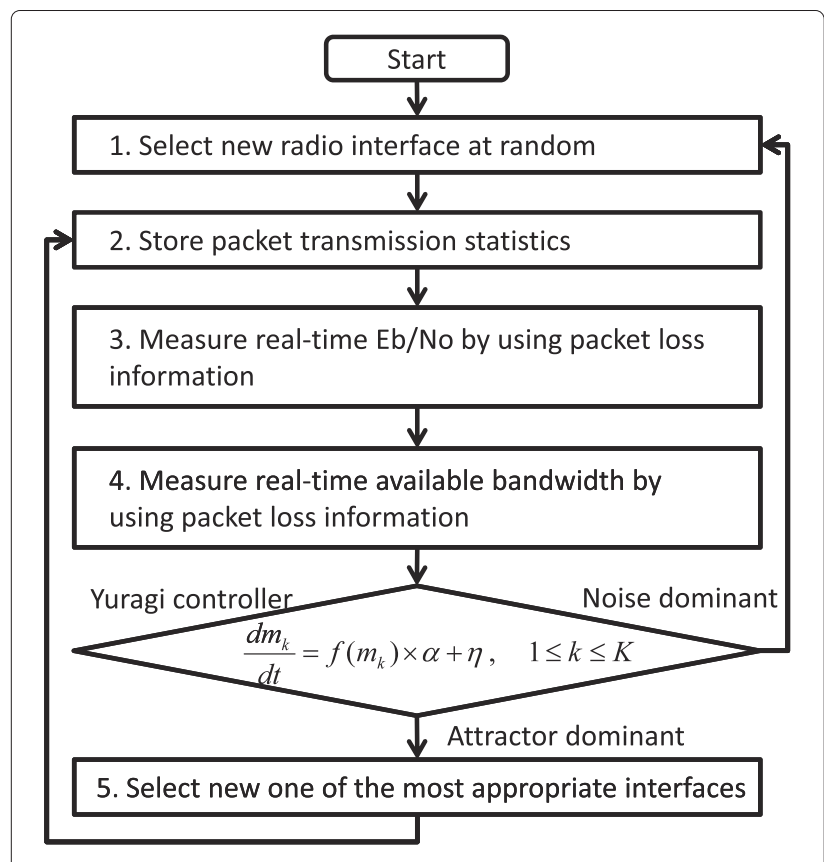

Figure 6 An example of Yuragi-based selection algorithm.

avoid unnecessary interface shifts, a hysteresis mechanism based on forward and backward protection is one of candidates to compensate for it. On the contrary, Figure $8 \mathrm{~d}$ shows that for $40 \mathrm{~dB}$ the initial noise level is so low that any noise level fluctuation does not produce any opportunities to shift the best interface and sticks to the radio interface \#0 to have an advantage of $10 \mathrm{~dB}$ after a transient stage. This is because noise fluctuation is not sufficient to drive the attractor selection mechanism properly. Apart from the above results, in Figure $8 \mathrm{c}$ it is observed that in case of $30 \mathrm{~dB}$ the selected best interface is adjusted according to the noise level shift caused by sudden degradation and recovery from the degradation. From these results, we confirm that our proposed mechanism is feasible enough for radio interface selection.

In addition, in Figure 9a-d transitions of realtime bit error rate (BER) are shown, which are the results in the case that AWGN is equivalent to $10,20,30$, and $40 \mathrm{~dB}$, respectively. From these results, both cases that the radio interface with better BER is always selected though the initially selected interface does not offer better BER all the time. From results of Figures 8 and 9 , an $F_{0 \text {,base of }}$ $40 \mathrm{~dB}$ or higher produces very low and stable BER that the system does not need to shift its interface. Once the system selects the better interface, environmental changes like temporary radio quality degradation of even $40 \mathrm{~dB}$ do not reach the level to induce interface shifts and therefore the system needs more time until an interface is switched. It is confirmed that our proposed mechanism works well from the viewpoint of not only adaptation to environmental change but also selection of better radio link quality. Autonomous management with attractor selection is so lightweight for vertical handover and does not require monitoring signal strength which causes heavy load and therefore we believe that these simulation results can be evidence for energy saving to some extent.

As a first step, we introduced simulation results with typical parameters in this article. However, simulations with well adjusted and wide variety of parameters have a potential to show more clarifying results in a later study.

\section{Numerical analysis of adaptive cluster selection}

In this section, numerical analysis for adaptive cluster selection is introduced. A network configuration is shown in Figure 10. In this figure, two OpenFlow networks are jointly connected in a hierarchical manner. Here, $\mathrm{CN}$, OFC, OFS, and BS indicate Correspondent Node, OFC, OpenFlow Switch, and Base Station, respectively. In addition, small numbers in this figure show the number of hops in message exchanges with reference to the previous cost calculation model for current 3G mobile systems [39]. In addition, basic parameters for cost analysis are shown in Table 2. The number of message hops for one inter-domain handover in Table 2 is calculated based on a typical inter-domain handover scenario shown in Figure 2.

The signaling cost incurred by inter-domain handover can be defined as:

$$
\text { Cost }=P \times S \times H
$$

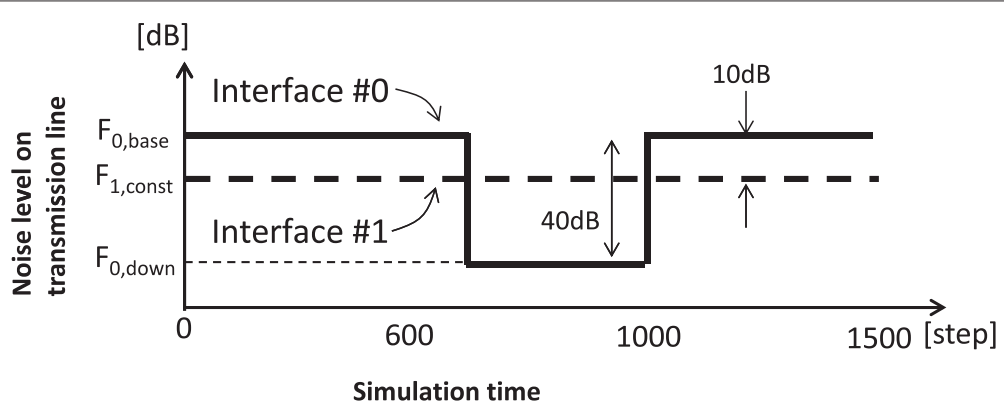

Figure 7 Scenario of radio quality changes. 


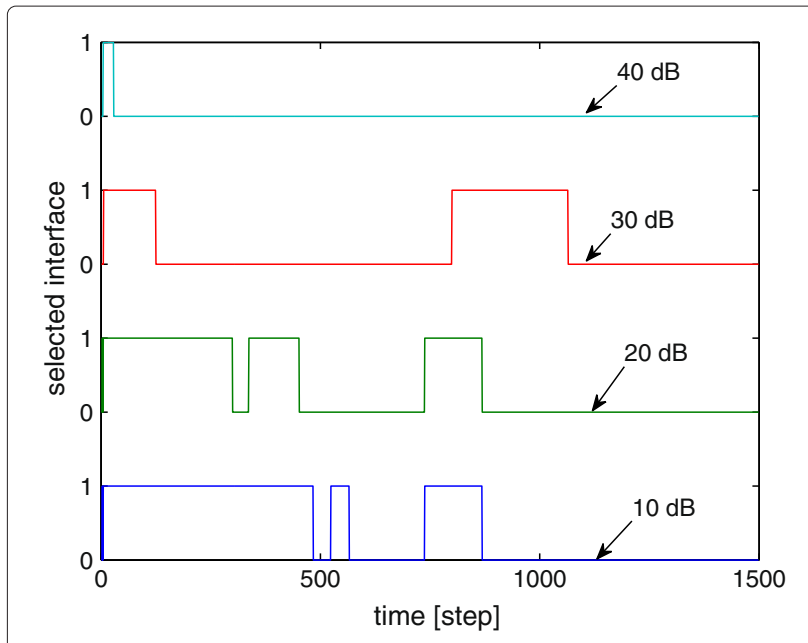

Figure 8 Selected interface change with different level of noises.

where $P$ is the probability that the handover occurs, $S$ is the average size of signaling sequence, and $H$ is the average number of hops for signal traversing. In addition, making use of concepts of network residence time $T_{n r}$ and session duration time $T_{s d}$, session arrival probability $P_{a}$ and interdomain handover occurrence probability $P_{h}$ are expressed as follows:

$$
\begin{aligned}
& P_{a}=\frac{\lambda \phi}{(\lambda+\phi)^{2}} \\
& P_{h}=\frac{\phi}{\mu+\phi}
\end{aligned}
$$

where $\lambda$ is the average session arrival rate following a Poisson process, $\phi$ is the inter-domain mobility rate when network residence time $T_{n r}$ is exponentially distributed with a mean $1 / \phi$, and $\mu$ is the average session service rate when session duration time $T_{s d}$ is exponentially distributed with a mean $1 / \mu$. Through the above discussion, the cost for inter-domain handover is expressed as follows

$$
\text { Cost }=P_{a} \lambda \sum_{i}^{n_{a}}\left(S_{a, i} \times H_{a, i}\right)+P_{h} \phi \sum_{k}^{n_{h}}\left(S_{h, k} \times H_{h, k}\right)
$$

where $S_{a}, S_{h}, H_{a}$, and $H_{h}$ are the message sizes and the number of hops for session arrivals and handovers, respectively. Here, $n_{a}$ and $n_{h}$ are the number of messages for session arrivals and handovers.

Numerical analysis results are shown in Figure 11. In this analysis, it is assumed that a single OFC takes care of 30 OFSs and the cost caused by inter-domain handover and the cost by continuously operating attractor selection are compared. As cost for attractor selection, the results (a)

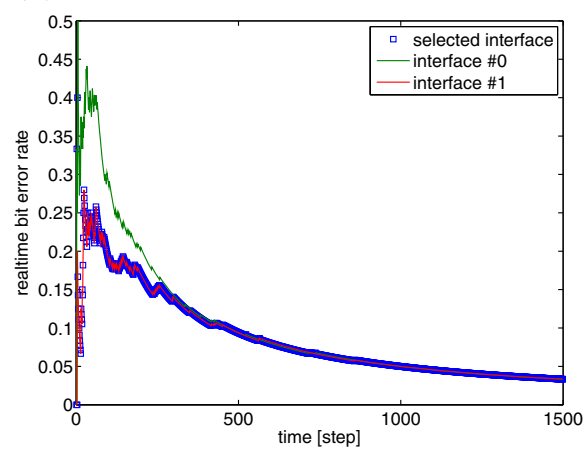

(c)

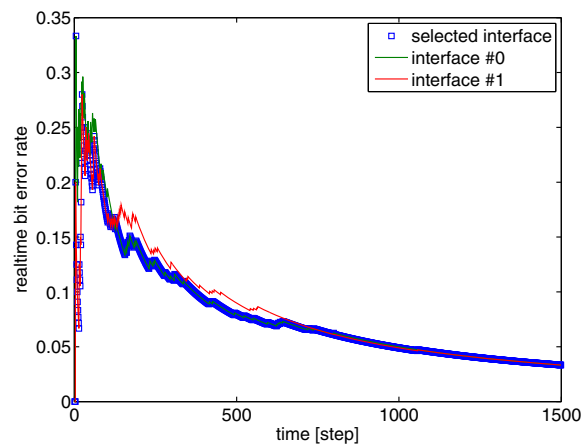

(b)

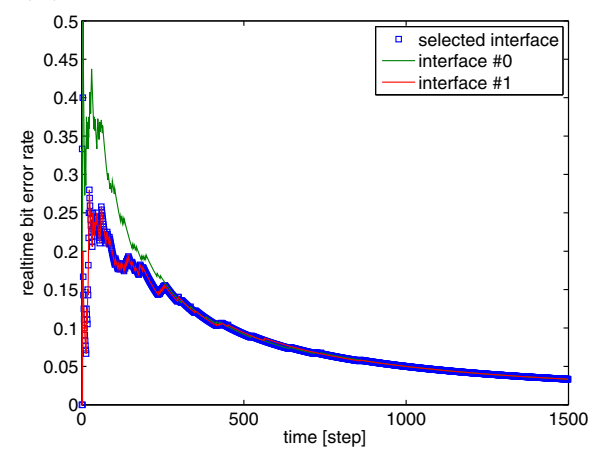

(d)

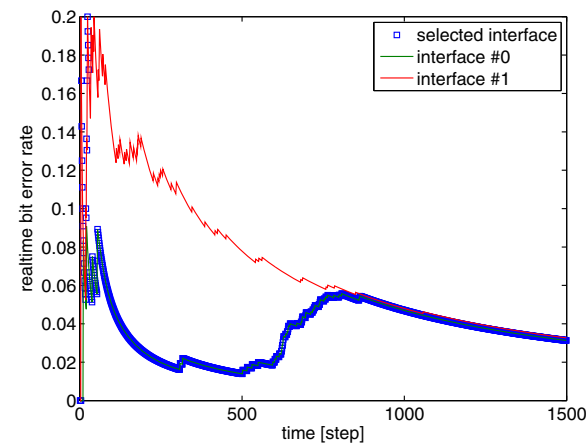

Figure 9 Realtime BER change with different level of noises. 


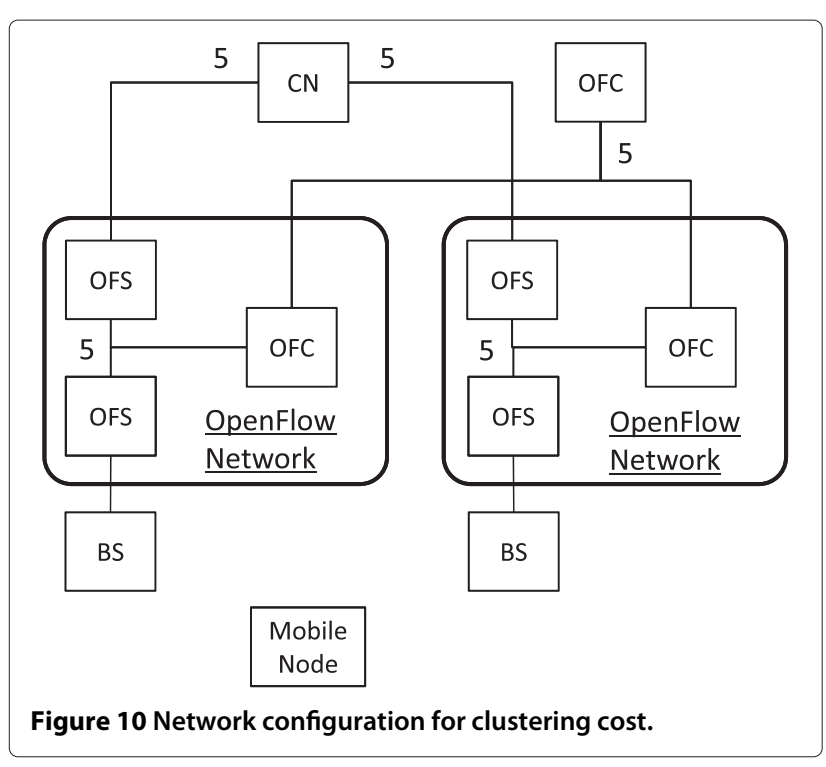

with unit processing time of once per second and once per minute are shown. In addition, the handover cost is calculated in Equation (12), which is the cost caused by message exchanges when an inter-domain handover is executed based on the sequence shown in Figure 2. In Figure 11, inter-domain mobility rate $\phi$ has different influence on session arrival rate and inter-domain handover probability in Equations (10) and (11). In addition, cost caused by inter-domain handover is dominant in higher network mobility rate and on the other hand, cost caused by session arrival rate is dominant in lower network mobility rate. Therefore, depending on relative relationship between session arrival rate and inter-domain handover probability, network mobility rate causes a tradeoff between the first and second sum on the right-hand side of Equation (12), which leads to the convex shape of the handover cost curve in Figure 11 with a minimal value. Here in this article, an unit of network mobility rate is the number of arrivals per minutes. From Figure 11, it is observed that at network mobility rate of 5 or more, handover cost becomes so high compared with the cost by attractor selection that attractor selection is more effective from cost viewpoint and more than five times higher cost reduction has been achieved at network mobility rate 10 .

Table 2 Basic parameters in adaptive clustering cost analysis

\begin{tabular}{ll}
\hline Parameter & Value \\
\hline Average packet length & 500 bytes $(=4000$ bits $)$ \\
Average message service rate & $0.00025(=1 / 4000)$ \\
Capacity of channel & 2 Mbps \\
Average session arrival rate & 0.001 \\
Number of message hops for one handover & 40 hops \\
\hline
\end{tabular}

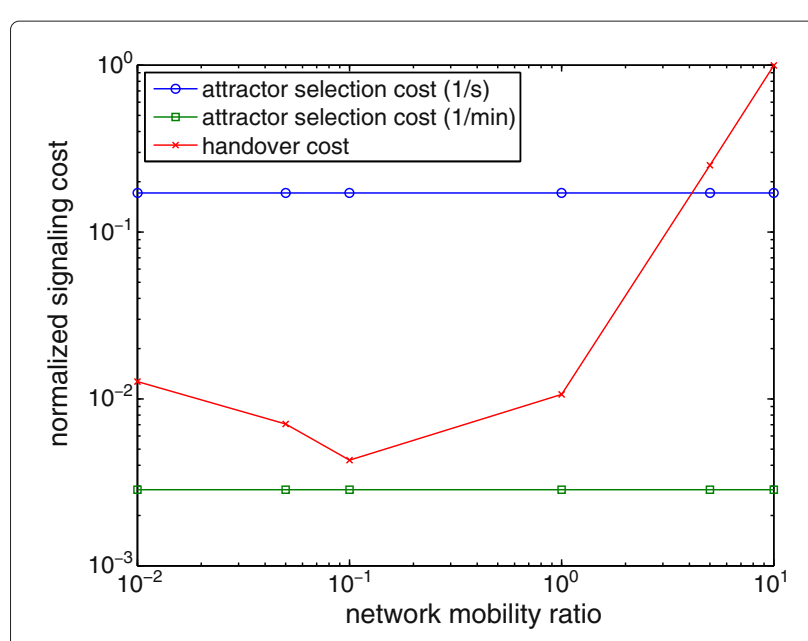

Figure 11 Numerical analysis results of handover cost.

In addition, at network mobility rate of less than 1, processing once per minute outperforms the handover cost and achieves about $75 \%$ cost reduction. However, processing once per second is higher than the handover cost and brings about four times cost increase. This cost reduction leads to energy saving from the viewpoint of processing cost. As a result, our proposed method is cost efficient compared with conventional ordinary inter-domain handover methods.

\section{Conclusion}

In this article, we discussed the future mobile network environment and the possibility of combining OpenFlow with the biologically inspired attractor selection method. In particular, we proposed two novel concepts of mobile network management based on the application of attractor selection.

First, we considered a concept to select the most appropriate interfaces for mobile nodes depending on instantaneous traffic volume. Instead of using preselected static information as a driving parameter of the attractor selection model as in [31], our proposed method uses realtime dynamic information and therefore, the controlling function can be more sensitively operated. In the end, the network resources are expected to be utilized in an effective way at the right place and at the right time. Second, a concept to build the most appropriate management domains was proposed. This is targeted to effectively reduce handover signaling cost. In the future, a wide variety of wireless access technologies will bring users more convenience, but bring operators additional management cost at the same time. Hence, we believe that our suggested concepts are feasible and could become one of the essential schemes for management cost reduction.

Our future work is to evaluate each method based on our concepts by greater variety of simulations to show 
advantages of our proposal and to formulate specific attractor selection models especially on issues for the future mobile network. In addition, a detailed quantitative analysis for energy saving is also our future work. Finally, we intend to confirm that they can work on a real system through a testbed implementation.

\section{Endnote}

a "Yuragi" is the Japanese expression for noise or fluctuations which is a key aspect in the attractor selection mechanism.

\section{Competing interests}

The authors declare that they have no competing interests.

\section{Author details}

${ }^{1}$ Cloud Systems Research Laboratories, NEC Corporation, 1753 Shimonumabe, Nakahara-ku, Kawasaki, Kanagawa, 211-8666, Japan. ${ }^{2}$ Graduate School of Information Science and Technology, Osaka University, 1-5 Yamadaoka, Suita, Osaka 565-0871, Japan. ${ }^{3}$ Center for Information and Neural Networks, National Institute of Information and Communications Technology, 1-3 Yamadaoka, Suita, Osaka 565-0871, Japan.

Received: 3 February 2012 Accepted: 25 July 2012

Published: 16 August 2012

\section{References}

1. J Pan, S Paul, R Jain, A survey of the research on future internet architectures. IEEE Commun. Mag. 49, 26-36 (2011)

2. NSF Future Internet Architecture Project, http://www.nets-fia.net/

3. Future Internet Assembly, http://www.future-internet.eu/home/futureinternet-assembly.html

4. AKARI Project: New Generation Network Architecture AKARI Conceptual Design (ver2.0 in Japanese and ver1.1 in English), http://akari-project.nict. go.jp/eng/overview.htm

5. MobilityFirst Future Internet Architecture Project, http://mobilityfirst. winlab.rutgers.edu/

6. J Pan, S Paul, R Jain, M Bowman, MILSA: a mobility and multihoming supporting identifier locator split architecture for naming in the next generation internet. in Proceedings of IEEE Global Communications, Conference (GLOBECOM'08) (New Orleans, 2008), pp. 1-6

7. Open Networking Foundation, http://www.openflow.org/

8. N McKeown, T Anderson, H Balakrishnan, G Parulkar, L Peterson, J Rexford, S Shenker, J Turner, OpenFlow: enabling innovation in campus networks. ACM SIGCOMM Comput. Commun. Rev. 38(2), 69-74 (2008)

9. KK Yap, R Sherwood, M Kobayashi, TY Huangy, M Chan, N Handigol, N McKeown, G Parulkar, Blueprint for introducing innovation into wireless mobile networks. in Proceedings of the 2nd ACM SIGCOMM International Workshop on Virtualized Infrastructure Systems and Architectures (VISA'10) (New Delhi, 2010), pp. 25-32

10. KK Yap, M Kobayashi, R Sherwood, TY Huang, M Chan, N Handigol, N McKeown, Openroads: empowering research in mobile networks. ACM SIGCOMM Comput. Commun. Rev. 40, 125-126 (2010)

11. S Balasubramaniam, K Leibnitz, P Lio, D Botvich, M Murata, Biological principles for future internet architecture design. IEEE Commun. Mag. (Special Issue on Future Internet Architectures: Design and Deployment Perspectives). 49(7), 44-52 (2011)

12. F Dressler, OB Akan, Bio-inspired networking: from theory to practice. IEEE Commun. Mag. 48(11), 176-183 (2010)

13. M Meisel, $\vee$ Pappas, $L$ Zhang, A taxonomy of biologically inspired research in computer networking. Comput. Netw. 54(6), 901-916 (2010)

14. Yuragi Project, http://www.yuragi.osaka-u.ac.jp/index.html

15. M Murata, Toward establishing ambient network environment. IEICE Trans. Commun. E92-B(4), 1070-1076 (2009)

16. J Mitola III, GQ Maguire, Cognitive radio: making software radios more personal. IEEE Personal Commun. 6(4), 13-18 (1999)
17. F Zhu, J McNair, Optimizations for vertical handoff decision algorithms. in Proceedings of the IEEE Wireless Communications and Networking Conference 2004 (WCNC'04), vol. 2 (Atlanta, 2004), pp. 867-872

18. S Merlin, N Vaidya, M Zorzi, Resource allocation in multi-radio multi-channel multi-hop wireless networks. in Proceedings of the 27th IEEE International Conference on Computer Communications (INFOCOMauthor='08) (Phoenix, 2008), pp. 610-618

19. M Kassar, B Kervella, G Pujolle, An overview of vertical handover decision strategies in heterogeneous wireless networks. Elsevier J. Comput. Commun. 31, 2607-2620 (2008)

20. S Yi, J Heo, Y Cho, J Hong, PEACH: power-efficient and adaptive clustering hierarchy protocol for wireless sensor networks. Elsevier J. Comput. Commun. 30, 2842-2852 (2007)

21. C Jiang, D Yuan, Y Zhao, Towards clustering algorithms in wireless sensor networks - a survey. in Proceedings of the IEEE Wireless Communications and Networking Conference 2009 (WCNC'09) (Budapest, 2009), pp. 1-6

22. G Di Caro, F Ducatelle, LM Gambardella, AntHocNet: an adaptive nature-inspired algorithm for routing in mobile ad hoc networks. Eur. Trans. Telecommun. 16(5), 443-455 (2005)

23. R Teng, K Leibnitz, B Zhang, Immune system inspired reliable query dissemination in wireless sensor networks. in Proceedings of the 10th International Conference on Artificial Immune Systems (ICARIS) (Cambridge, 2011), pp. 282-293

24. S Balasubramaniam, D Botvich, J Mineraud, W Donnelly, N Agoulmine, BiRSM: bio-inspired resource self-management for all IP-networks. IEEE Netw. 24(3), 20-25 (2010)

25. I Carreras, I Chlamtac, H Woesner, C Kiraly, BIONETS: bio-inspired next generation networks. Auton. Commun. 3457, 273-274 (2005)

26. J Suzuki, T Suda, A middleware platform for a biologically inspired network architecture supporting autonomous and adaptive applications. IEEE J. Sel. Areas Commun. 23(2), 249-260 (2005)

27. A Kashiwagi, I Urabe, K Kaneko, T Yomo, Adaptive response of a gene network to environmental changes by fitness-induced attractor selection. PLoS ONE. 1 (e49) (2006)

28. K Leibnitz, N Wakamiya, M Murata, Biologically-inspired self-adaptive multi-path routing in overlay networks. ACM Commun. (Special Issue on Self-Managed Systems and Services). 49(3), 62-67 (2006)

29. N Wakamiya, K Leibnitz, M Murata, A Self-Organizing Architecture for scalable, adaptive, and robust networking. in Autonomic Network Management Principles: From Concepts to Applications, ed. by N Agoulmine. (Elsevier, 2010), pp. 119-140

30. K Leibnitz, M Murata, Attractor selection and perturbation for robust networks in fluctuating environments. IEEE Netw. (Special Issue on Biologically Inspired Networking). 24(3), 14-18 (2010)

31. S Kajioka, N Wakamiya, M Murata, Autonomous and adaptive resource allocation among multiple nodes and multiple applications in heterogeneous wireless networks. Elsevier J. Comput. Syst. Sci (2011) in press

32. Y Koizumi, T Miyamura, S Arakawa, E Oki, K Shiomoto, M Murata, Adaptive virtual network topology control based on attarctor selection. IEEE J. Lightwave Technol. 28(11), 1720-1731 (2010)

33. $\mathrm{YLi}, \mathrm{J}$ Wang, $\mathrm{Y} \mathrm{Xu}$, Adaptive intentional connection rerouting for traffic grooming in WDM mesh networks with biological attractor selection. in Proceedings of the 16th Asia-Pacific Conference on Communications (APCC'10) (Auckland, 2010), pp. 83-88

34. H Chujo, H Oka, Y Ikkai, N Komoda, A real-time production scheduling method using attractor selection. in Proceedings of the International Conference on Computational Intelligence for Modelling, Control and Automation, and International Conference on Intelligent Agents, Web Technologies and Internet Commerce (CIMCA-IAWTIC'05) (Vienna, 2005), pp. 511-516

35. I Fukuyori, Y Nakamura, Y Matsumoto, H Ishiguro, Control method for a robot based on the adaptive attractor selection model. in Proceedings of the 4th International Conference on Autonomous Robots and Agents (ICARA'09) (Wellington, 2009), pp. 618-623

36. S Kitajima, THara, T Terada, S Nishio, Filtering order adaptation based on attractor selection for data broadcasting system. in Proceedings of the International Conference on Complex, Intelligent and Software Intensive Systems (CISIS'09) (Fukuoka, 2009), pp. 319-326

37. WC Jakes, Microwave Mobile Communications (IEEE Press, New York, 1994) 
38. V Chandrasekhar, JG Andrews, A Gatherer, Femtocell networks: a survey. IEEE Commun. Mag. 46(9), 59-67 (2009)

39. KS Munasinghe, A Jamalipour, Analysis of signaling cost for a roaming user in a heterogeneous mobile data network. in Proceedings of IEEE Global Telecommunications, Conference (GLOBECOM'08) (New Orleans, 2008), pp. 1-5

\section{doi:10.1186/1687-1499-2012-259}

Cite this article as: Motoyoshi et al:: Proposal and evaluation of a future mobile network management mechanism with attractor selection. EURASIP Journal on Wireless Communications and Networking 2012 2012:259.

\section{Submit your manuscript to a SpringerOpen ${ }^{\circ}$ journal and benefit from: \\ - Convenient online submission \\ - Rigorous peer review \\ - Immediate publication on acceptance \\ - Open access: articles freely available online \\ - High visibility within the field \\ - Retaining the copyright to your article \\ Submit your next manuscript at $\boldsymbol{\wedge}$ springeropen.com}

